\title{
OCEAN WAVE PHYSICS AND MODELING The Message from the 2019 WISE Meeting
}

\author{
Luigi Cavaleri, Francesco Barbariol, Alvise Benetazzo, and Takujı Waseda
}

W ithin the present abundance of ocean wave physics and modeling meetings, Waves in Sea Environment (WISE) still keeps the original [from the Wave Model (WAM) meetings] purpose of a collective and open discussion of where the wave community is and wants to go. Although the increased popularity has now transcended the original purpose of a purely working workshop, the number of attendees is still limited enough to allow continuous discussion, helped also by the extended poster sessions full of personalized debates. WISE, held on a yearly basis, provides a rather general perspective of the present active branches of wave research. In this paper, we provide a compact list of, and comments on, the most recent results and the expected future developments. Following its original spirit of pure discussion, no written documentation is produced. The program of the meeting, ${ }^{1}$ with authors and titles, is available at www.orca.k.u-tokyo.ac.jp /WasedaLab/wise2019/. The interested reader may approach the individual authors for specific information.

AfFiliations: CaValeri, Barbariol, and Benetazzo-Institute of Marine Sciences, National Research Council, Venice, Italy; WASEDA-The University of Tokyo, Tokyo, Japan CORRESPONDING AUTHOR: Luigi Cavaleri, luigi.cavaleri@ismar.cnr.it

DOI:I0.II75/BAMS-D-19-0195.I

In final form 13 September 2019 (C)2019 American Meteorological Society For information regarding reuse of this content and general copyright information, consult the AMS Copyright Policy.
WISE: WAVES IN SEA ENVIRONMENT
WHAT: Eighty wind-wave scholars from 22 countries met to report on their latest results, both as applications and wave physics, discussing not only the present state of the art, but mainly the lines along which future actions, in particular the interactions between ocean and atmosphere, should be directed.
WHEN: 12-16 May 2019
WHERE: Jozankei, Hokkaido, Japan

PHYSICAL PROCESSES. When it comes to modeling waves in a marine storm or, at the other extreme, swell propagating on the expanse of the oceans, the physical-based approach is still at the heart of any wave model. Out of the three basic source functions of the wave action equation, energy dissipation via breaking (white-capping) is presently the focus of most research. The reason, as we will see in the Modeling section, is its fundamental role in coupling the ocean surface with the lower atmosphere (spray, bubbles, strongly increased contact surface between the two media, bursts of momentum from air to sea and from waves to current, $30 \%$ increased drift with respect to the pure Stokes model). Besides, white-capping is still considered the least-known physical term of the equation, often used as the main tuning knob to get the correct energy balance. The recently proposed ST6 source-term package of the

\footnotetext{
${ }^{1}$ The 2019 meeting was locally organized by Takuji Waseda and his team at The University of Tokyo.
} 
WAVEWATCH III wave model aims at largely limiting any user action on the source terms. However, intense interest in why and when a single wave crest breaks strongly suggests that the final solution for phase-averaged spectral models is not yet at hand.

Nonlinear wave-wave interactions have been for a long while the focus of much work and attention, but they seem to have now lost their spin. Alternatives to the much-criticized but heavily used, for practical purposes, Discrete Interaction Approximation (DIA) approach are well known, but they are mostly shelved in operational applications because of their much higher computational cost. Computer power and speed have increased orders of magnitude since the first alternative was produced. However, with each improvement the choice has been to favor grid resolution (with the implied limitations of the integration time step). Possibly, with the present unstructured grids and the "best" resolution now achieved, future attention will go back to nonlinear interactions. The problem will still persist for the most intense storms in the ocean, however.

A still-debated subject is the role of wave orbital motion in deep sea mixing. The community is divided in this respect, looking for a possible alternative interpretation such as the concept of "Langmuir turbulence." However, more orthodox approaches still fail to reach the correct depth of the ocean mixed layer.

A very theoretical school dealing with our main subject of interest-the basic source functions of the energy equation-keeps providing brilliant solutions to theoretical problems, in principle, but not in practice. The problem is that we have not yet found a way to apply these findings daily to our real world.

EXPERIMENTS. With respect to the open sea measurements (next section), we refer here to the measurements done in a controlled environment, typically a laboratory or a wave tank. This allows repeated, controlled, and selected experiments with a specific purpose in mind. Strongly increased technical capabilities now allow a focus on tiny details, as the very first stage of (fetch limited and transient) wave generation by wind. Often this leads to making previously unsuspected details evident despite still lacking an explanation. Examples of this are the two clearly different stages of very early wave growth (possibly two different processes at work) or the presence of minute corrugations on the forward face of the very short wavelets. It is fair to say that in wave modeling these are self-standing problems, in that in practical applications these very early stages are bypassed, for example, accepting at each time step as a minimum energy the one corresponding to one-grid-step, fetchlimited JONSWAP (Joint North Sea Wave Project) spectrum (of course function of the local wind).

High-resolution recording in time and space has visualized one of the key processes for spray formation in a breaking wave. Under the action of wind the wave upper border evolves into a series of minute and unstable thin, curved water layers (sails). Lasting a fraction of a second, their breaking leads to the formation of minute drops that are a substantial part of the surface spray typical of active sea conditions. These sails provide a beautiful example of how much we need to dig in to really understand what is going on.

Suitably prepared experiments in the laboratory, or better a wind wave tank, can be used to derive general results on wind-wave interactions. In particular, the presence of active surface agents (an almost monomolecular layer of fish oil) has allowed for the first time the visualization of the effects of the pure frictional turbulent wind stress. This line of thinking is expected to lead to more basic results in the future.

Much attention has been recently given to waves and ice interactions. We report on this in the Ice section.

MEASUREMENTS. Wave data have long been the basic information to know about the sea and what was going on. Nowadays they are still used for the same purpose, but largely to validate our model results. Apart from a limited number of on-rig measuring systems, the workhorse of open ocean data collection has been the buoy. Considered more reliable than satellite data (hence used for their calibration), wave buoys are in a transition period. Looking for (production and management) cheaper solutions, allowed for by technological advances, buoys are becoming smaller and lighter. This may sound positive, but this will require a new assessment of how each buoy reacts to wave motion, implicitly of its calibration, to provide meaningful and accurate wave data.

The economical possibility of using many small drifting buoys for a specific purpose provides the opportunity for devoted experiments. Recording swell along the California coast has revealed how its characteristics, in particular specific local values but more so the width of its directional distribution, are in general varying more and wider than expected by geometrical optics approximation. This phenomenon is defined as "scintillation." The explanation, theoretically formulated, then verified by data, is that swell is refracted by many small-scale current structures, which on average and across many interactions will tend to broaden the directionality of the wave field. In 
the present fully coupled operational models (see next section) only the large-scale currents are considered.

Long-term buoy data are probably the most reliable source for extremes estimates, for long-term statistics. While the buoys are relatively limited in number and mostly are not far from the coast, the uniform characteristics of the instrument throughout the time series are key for long-term estimates. This takes us back to the abovementioned change of the buoy hulls and hardware. "Real" buoy behavior, especially in rough sea conditions, is in question. Moored buoys are well known for skirting higher and steeper crests. The question is, is there a better instrument for measuring waves? The answer is positive: the videostereo system. Recently perfected, hardware and open-access software provide, from a fixed support, a phase-resolving ( $2 \mathrm{D}+$ time) view of a large enough section of the sea surface elevation to derive the actual wave motion, at centimetric resolution. Very accurate estimates of 3D wavenumber-frequency spectra and much-improved statistics of the sea surface have been derived, including extremes and freak waves and their impacts on offshore structures, all this also in extreme conditions where the use of buoys could be problematic. The system has two limitations: the need for a fixed support (a pole, or a rig, or similar) and the capability of working only during daylight. However, such a system can be, and has successfully been, used to monitor the motion of a buoy, fully defining its behavior in a more or less rough sea.

Satellite data, in particular from Synthetic Aperture Radar (SAR) and altimeters, keep providing tremendous amounts of wave data. The recently launched, and soon to be open to the community, Chinese-French Oceanography Satellite (CFOSAT) brings high expectations. The actual accuracy of the data is still to be assessed. As an example, the European Space Agency (ESA) recently announced a project for a new analysis of all past altimeter data to correct existing historical estimates.

MODELING. The dominant image in the mind of a wave modeler is "the wave," the physical elevation and motion of the sea surface that was the only target of the first generation of wave modelers. However, it is now clear that atmosphere and ocean are tightly coupled, one affecting the other's behavior, continuously exchanging physical "information." Because all these exchanges pass through the ocean surface, they are strongly affected by its characteristics, that is, by the wave field. This is to stress that, while a first-order estimate of a storm can be obtained via only windto-waves information, any correct estimate, and not only for wave purposes, requires two-way coupled modeling. This is the way to go, and indeed it is the one followed by the major operational forecast centers.

This approach implies a better definition of the processes at the interface. These can be approached theoretically or with reasoned parameterizations, both at large and subgrid scales. However, particularly at the smallest scales and in the most extreme cases, the physics may defy imagination, and devoted physical (as in the Experiments section) or computational experiments are required. Computational fluid dynamics (CFD) is particularly suited for these tests, and indeed it provides beautiful and (at first glance) realistic representations of the spray production at very high wind speeds.

One of the key questions is still which part of the wind stress goes into turbulent stress and which one into the wave system, which is then split between wave growth and white-capping? As in the previous section, devoted experiments may yield the response.

While we look for the best solution, we still have to solve the daily problem of the most realistic wave forecast. Therefore "engineering solutions," as empirically correcting for known model deficiencies, are still acceptable. A typical case is how the model performance depends on air-sea stability conditions. However, these patches are (coupled) model dependent, and they should be only a temporary solution.

Approaching the coasts, model resolution needs to be increased, and the most efficient approach is to use unstructured grids. Resolution can go down from what is desired offshore to $10 \mathrm{~m}$ close to the coast. At this resolution, all the corresponding necessary information (wind and bathymetry accuracy to start with) must also be available. The effort makes sense if we go into more details with the correct local physics. We need to have clear in our mind what we are actually looking for.

Long-term atmosphere-ocean-wave reanalyses are providing information for many studies all around the globe. ERA-Interim and the recently released ERA5 (both from ECMWF) provide unprecedented volumes of data, including directional wave spectra, throughout the world. Presently available since 1979, it will soon be extended back to 1950 . The availability of reanalysis wave spectra has allowed a reliable estimate of the expected extreme and freak waves at any location throughout the globe (under present climate conditions). However, the use of spectra allows us to define how partitions (in practice the different wave systems) vary in time and at any location. In so doing we can study their evolution, and in practice the trends of the wave climate. 
ICE. The shrinking Arctic ice extent has triggered much political and economic interest, and as a consequence has increased scientific attention. For waves, there are two main lines of research. One is the physical interaction among waves and ice floes in the marginal ice zone. The problem is approached in several different ways: 1) putting instruments in the sea and on ice in the field; 2) working with paddle waves impinging on a rubber sheet simulating a floe, or on ice cubes, in the laboratory; and 3) working with CFD. The purpose is to see how much wave energy is attenuated crossing a floe field. A more interesting aspect is that these floes, as also the still liquid, but viscous stage of ice formation known as grease ice, kill the high-frequency tail of the spectrum. This changes, and drastically limits, the interactions between air and sea, in a way similar to pouring oil in an otherwise rough sea.

The second line of polar research concerns the estimate of the heights of the now-possible waves in the high-latitude seas. The reduced ice extent implies a wider open sea, longer fetches, stronger air-sea interactions, and in practice higher waves. Estimates for a fully free summer ocean are being produced. Note (more a subject for wave modeling) that dealing with the polar area may require quite a bit of numerical attention. A non-trivial problem is the substantial lack of data (also by satellite) in the area.

GENERAL CONSIDERATIONS. Wave modeling performance can be considered as following an asymptotic improvement. Apart from white-capping, where work is still going on, we lack new ideas about physics, and we are progressively moving toward large scale and time production. In a way, this makes sense because our results, when compared to measured data, are more than satisfactory. However, we are still unable to deal at the same level with extremes, and this suggests that our physics may not be as correct as we like to think. For instance, there are simmering doubts, not happily received, that wave generation based on the spectral approach may not represent the truth. Why do we accept wind-wave generation as formulated tens of years ago and argue instead about white-capping? Granted a different solid background, a reason can be that white-capping is a physically visible process, but we cannot "see" generation, and this makes for a more difficult argument. In any case, we lack the drastic advances, at least the theoretical ones. Certainly, we have them in technology, both in the laboratory and in the field-in the latter with the advent of video-stereo systems.

The improvements in the laboratories, especially at the minute scale, are somehow in contrast with the abovementioned large-scale production. The question is how to bring into the operational models the implications of the tiny physics we are now able to see.

Our last comment is on the way to the future. Ignoring for a moment all the specific subjects and problems we mentioned, it is clear that the hot topic for years to come is "coupling." This will imply abandoning any sectorial view and working with an open mind, embracing the problems that were once counterpart. Atmosphere and ocean must be considered as a whole system, interfaced by a wavy surface that controls all the exchanges between the two media. The subject we started with as an interesting, selfstanding phenomenon has revealed itself as one of the keys that control the evolution of the atmosphere and ocean climate. 